\title{
Ärztliche Weiterbildung auf dem Prüfstand
}

\author{
Erste Ergebnisse der aktuellen Umfrage bei Assistenzärztinnen und -ärzten über die Weiterbildung
}

Max Giger, Ressort Medical Education, FMH

Michael Siegrist, Sozialforschungsstelle, Universität Zürich

$67 \%$ der Assistenzärztinnen und Assistenzärzte, die ihre Weiterbildung zum Facharzttitel absolvieren, beantworteten zwischen August und Oktober 2003 die Fragen zur Qualität der Weiterbildung. Es handelt sich um $47 \%$ Frauen und 53\% Männer. Knapp ein Viertel aller Assistenzärztinnen und Assistenzärzte stammt aus Deutschland. Die Weiterbildung wird von 14,6\% der Frauen und von 3,8\% der Männer in Teilzeit absolviert. Die Weiterbildung kann von der Hälfte der Antwortenden nicht zu ihrer Zufriedenheit innerhalb der normalen Arbeitszeit absolviert werden. Die Antworten auf die diesjährigen Zusatzfragen weisen auf einen ungenügenden Praxisbezug der Ausbildung hin.

Die Umfrage zur Weiterbildung unter den Assistenzärzten erfolgte in den Monaten August bis Oktober 2003 mit einem neuen Fragebogen. Dieser wurde aufgrund der früheren Erfahrungen und den Erkenntnissen von Workshops mit Weiterbildnern und Assistenzärzten erarbeitet und an zwei universitären Zentren ausgetestet. Es wurden Fragen in neun für die Weiterbildung relevanten Feldern, zusätzliche Fragen zu den persönlichen Kosten der Weiterbildung, zur Ausbildung und zur Person gestellt. Die Verantwortlichen der Weiterbildungsstätten erhalten einen detaillierten Bericht über die Beurteilung der neun relevanten Felder durch ihre Assistenzärzte. Im folgenden werden aggregierte Daten über die Weiterbildungsaktivitäten und ausgewählte Fragen zum Prozess präsentiert.

1191 Leiter von Weiterbildungsstätten haben den Fragebogen an 8001 Assistenzärzte weitergeleitet. Die Rücklaufquote beträgt 67\% (5343) und liegt $7-10 \%$ tiefer als in den sechs vorausgegangenen Umfragen. 47\% der Fragebogen sind von Assistenzärztinnen, 53\% von Assistenzärzten ausgefüllt worden. Von 103 Weiterbildungsstätten wurde aus unbekannten Gründen kein Fragebogen zurückgesandt. 14,6\% der Frauen und 3,8\% der Männer absolvieren ihre Weiterbildung in Teilzeit. Der Ort des Arztdiploms ist in Tabelle 1 dargestellt.

Die Antworten auf die Frage nach dem angestrebten Facharzttitel sind in Tabelle 2 dargestellt. Auffallend ist die starke Feminisierung von Anästhesiologie, Gynäkologie, Kinder- und Jugendmedizin und Kinder- und Jugendpsychiatrie.
Tabelle 1

Herkunft der Assistenzärzte (Ort des Arztdiploms). In welchem Land haben Sie das Staatsexamen abgeschlossen?

\begin{tabular}{lrr} 
& Zahl & $\%$ \\
\hline $\mathrm{CH}$ & 3561 & 67,8 \\
\hline $\mathrm{D}$ & 1187 & 22,6 \\
$\mathrm{~F}$ & 30 & 0,6 \\
$\mathrm{I}$ & 93 & 1,8 \\
\hline $\mathrm{A}$ & 69 & 1,3 \\
\hline restliche EU & 74 & 1,4 \\
\hline anderes Land & 239 & 4,5 \\
\hline Total & 5253 & 100,0
\end{tabular}

Für die Beurteilung der Weiterbildungsstätten wurden den Befragten Aussagen vorgelegt. Die Befragten konnten eine von 6 vorgegebenen Antwortkategorien auswählen. Von den Antwortkategorien waren nur die beiden Extrempunkte 1 (trifft überhaupt nicht zu) und 6 (trifft voll und ganz zu) verbal verankert. Aufgrund von inhaltlichen und statistischen Überlegungen wurden die Fragen in neun Skalen oder Themenfelder zusammengefasst, welche für die Bewertung einer Weiterbildungsstätte zentral sind. Aus Fragen, die hoch miteinander korrelierten, wurden Skalenwerte gebildet. Die Skalen weisen kleinere Messfehler auf als die einzelnen Fragen. Dadurch sind zuverlässigere Aussagen möglich. Die gebildeten Skalen weisen insgesamt eine hohe Messgenauigkeit auf. Die Durchschnittswerte für alle Personen, welche den Fragebogen ausfüllten, sind in einem Übersichtsdiagramm (Abb. 1) dargestellt.

Eher tiefe Werte können für die Fehlerkultur und für die Evidence-based medicine beobachtet werden. In diesen Bereichen ist ein noch unausgeschöpftes Verbesserungspotential vorhanden. Gute Werte konnten für die Lernkultur und die Betriebskultur beobachtet werden.

Die Mehrheit der Assistenzärzte kann zwar die Arbeit, nicht jedoch die Weiterbildung zu ihrer Zufriedenheit innerhalb der regulären Arbeitszeit erledigen (Abb. 2). 
Tabelle 2

Angestrebter Facharzttitel getrennt nach Geschlecht.

Welchen Facharzttitel streben Sie an?

\begin{tabular}{|c|c|c|c|c|}
\hline \multirow[t]{2}{*}{ Geschlecht } & \multirow{2}{*}{$\frac{\mathrm{m}}{\mathrm{Zahl}}$} & \multicolumn{3}{|c|}{ w } \\
\hline & & $\%$ & Zahl & $\%$ \\
\hline Allergologie und klinische Immunologie & 10 & 0,4 & 4 & 0,2 \\
\hline Allgemeinmedizin & 234 & 9,1 & 210 & 9,4 \\
\hline Anästhesiologie & 149 & 5,8 & 176 & 7,8 \\
\hline Angiologie & 14 & 0,5 & 2 & 0,1 \\
\hline Arbeitsmedizin & 8 & 0,3 & 7 & 0,3 \\
\hline Chirurgie & 202 & 7,8 & 87 & 3,9 \\
\hline Dermatologie und Venerologie & 31 & 1,2 & 37 & 1,6 \\
\hline Endokrinologie-Diabetologie & 11 & 0,4 & 12 & 0,5 \\
\hline Gastroenterologie & 24 & 0,9 & 11 & 0,5 \\
\hline Gynäkologie und Geburtshilfe & 54 & 2,1 & 275 & 12,3 \\
\hline Hämatologie & 14 & 0,5 & 12 & 0,5 \\
\hline Herz- und thorakale Gefässchirurgie & 16 & 0,6 & 2 & 0,1 \\
\hline Infektiologie & 23 & 0,9 & 14 & 0,6 \\
\hline Innere Medizin & 434 & 16,9 & 387 & 17,3 \\
\hline Intensivmedizin & 32 & 1,2 & 18 & 0,8 \\
\hline Kardiologie & 87 & 3,4 & 10 & 0,4 \\
\hline Kiefer- und Gesichtschirurgie & 15 & 0,6 & 1 & 0,0 \\
\hline Kinderchirurgie & 20 & 0,8 & 13 & 0,6 \\
\hline Kinder- und Jugendmedizin & 93 & 3,6 & 207 & 9,2 \\
\hline Kinder- und Jugendpsychiatrie und -psychotherapie & 37 & 1,4 & 117 & 5,2 \\
\hline Klinische Pharmakologie und Toxikologie & 4 & 0,2 & 6 & 0,3 \\
\hline Medizinische Genetik & 4 & 0,2 & 4 & 0,2 \\
\hline Medizinische Onkologie & 21 & 0,8 & 22 & 1,0 \\
\hline Nephrologie & 19 & 0,7 & 12 & 0,5 \\
\hline Neurochirurgie & 27 & 1,0 & 7 & 0,3 \\
\hline Neurologie & 55 & 2,1 & 41 & 1,8 \\
\hline Nuklearmedizin & 10 & 0,4 & 2 & 0,1 \\
\hline Ophthalmologie & 61 & 2,4 & 45 & 2,0 \\
\hline $\begin{array}{l}\text { Orthopädische Chirurgie und Traumatologie } \\
\text { des Bewegungsapparates }\end{array}$ & 190 & 7,4 & 35 & 1,6 \\
\hline Oto-Rhino-Laryngologie & 45 & 1,7 & 18 & 0,8 \\
\hline Pathologie & 29 & 1,1 & 30 & 1,3 \\
\hline Pharmazeutische Medizin & 15 & 0,6 & 7 & 0,3 \\
\hline Physikalische Medizin und Rehabilitation & 23 & 0,9 & 17 & 0,8 \\
\hline Plastische, rekonstruktive und ästhetische Chirurgie & 28 & 1,1 & 19 & 0,8 \\
\hline Pneumologie & 26 & 1,0 & 3 & 0,1 \\
\hline Prävention und Gesundheitswesen & 6 & 0,2 & 8 & 0,4 \\
\hline Psychiatrie und Psychotherapie & 329 & 12,8 & 281 & 12,5 \\
\hline Radiologie & 74 & 2,9 & 40 & 1,8 \\
\hline Radio-Onkologie / Strahlentherapie & 12 & 0,5 & 10 & 0,4 \\
\hline Rechtsmedizin & 14 & 0,5 & 9 & 0,4 \\
\hline Rheumatologie & 23 & 0,9 & 16 & 0,7 \\
\hline Tropen- und Reisemedizin & 2 & 0,1 & 1 & 0,0 \\
\hline Urologie & 49 & 1,9 & 8 & 0,4 \\
\hline Total & 2574 & 100,0 & 2243 & 100,0 \\
\hline
\end{tabular}

Bei der Ausarbeitung dieses Fragebogens wurde beschlossen, jedes Jahr ein zusätzliches Feld der medizinischen Aus- und Weiterbildung zu beleuchten. In diesem Jahre wurde die Frage nach der Praxisrelevanz der Ausbildung gestellt. Fünf Fragen wurden benutzt (z.B. «Der im Studium vermittelte Stoff ist praxisrelevant»). Die Antworten auf die fünf Fragen korrelierten hoch, es konnte deshalb eine Skala gebildet werden. Die möglichen Werte liegen zwischen 1 und 6. Ein hoher Wert bedeutet dabei, dass die Praxisrelevanz als hoch eingestuft wurde und ein tiefer Wert bedeutet, dass die Praxisrelevanz als ungenügend eingestuft wurde. Die Mittelwerte für die fünf Universitäten sind in Tabelle 3 dargestellt. Die Werte für die Universitäten Basel und Lausanne unterscheiden sich nicht signifikant, dasselbe gilt für die Universitäten Genf und Bern. Alle anderen Unterschiede sind statistisch bedeutsam.

\section{Beurteilung der Resultate und Ausblick}

Der neue Fragebogen erlaubt zum ersten Mal eine epidemiologische Beurteilung der Weiterbildung. Die Weiterbildung wird insgesamt als gut beurteilt. Dass rund ein Drittel der Antwortenden die Weiterbildung als ungenügend strukturiert beurteilt, ist ein Hinweis auf Verbesserungsmöglichkeiten in der theoretischen und praktischen Weiterbildung: «learning by doing» genügt nicht. Die Weiterbildungsinhalte sollten vermehrt strukturiert über eine bestimmte Zeiteinheit vermittelt werden. In der Weiterbildung müssen Fehlerkultur und Evidence-based medicine stärker gewichtet werden. Sind Fehlerkultur und Evidence-based medicine expliziter Bestandteil der Weiterbildung, darf eine raschere Verbreitung dieser für die Qualität der Patientenbetreuung wichtigen Instrumente erwartet werden. Der hohe Anteil von Deutschen in der Weiterbildung könnte entweder Ausdruck sein der strapazierten Personalsituation im ärztlichen Sektor vieler Spitäler oder der Attraktivität der Schweizer Weiterbildungsstätten.

Die im Vergleich zu früheren Umfragen tiefere Rücklaufquote kann zum Teil auf den grösseren Umfang des Fragebogens, zum Teil auf die fehlende Unterstützung durch Weiterbildner, zum Teil auf fehlende Motivation zur Teilnahme an der Umfrage zurückgeführt werden. Es muss daran gearbeitet werden, dass die Assistenzärzte die Beurteilung als selbstverständlichen Beitrag zur Verbesserung der Weiterbildung wahrnehmen. 
Tabelle 3

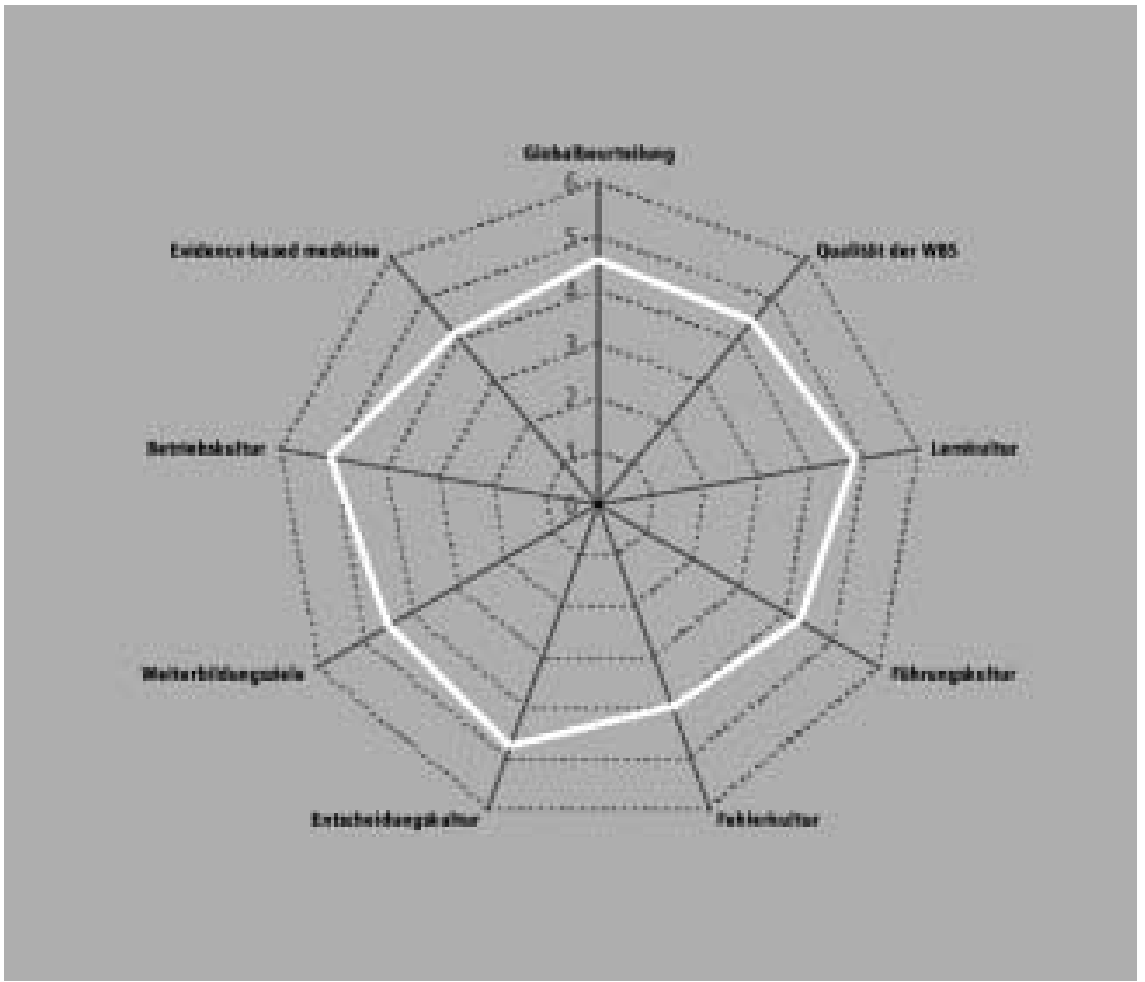

Praxisrelevanz der Ausbildung.

\begin{tabular}{llr} 
Universität & Mittelwert & $\begin{array}{r}95 \% \text { Konfidenz- } \\
\text { intervall }\end{array}$ \\
\hline Zürich & 2,77 & $2,72-2,83$ \\
\hline Basel & 2,97 & $2,89-3,04$ \\
\hline Lausanne & 3,09 & $3,01-3,17$ \\
\hline Genf & 3,46 & $3,36-3,55$ \\
\hline Bern & 3,59 & $3,51-3,66$
\end{tabular}

Die Resultate der Umfrage bestätigen den immer wieder gehörten Vorwurf an die universitäre Ausbildung, nämlich der ungenügenden Praxisrelevanz. Massnahmen zur Verbesserung sind in Form des Schweizerischen Lernzielkataloges jetzt an allen Universitäten eingeleitet. Vielleicht könnten die diesjährigen Fragen in fünf Jahren den Absolventen des reformierten Studiums gestellt werden?

Die Erfahrungen mit dem neuen Fragebogen sollen in einem Workshop mit den Leitern der Weiterbildungsstätten diskutiert werden und in die Umfrage des Jahres 2004 einfliessen. Eine detaillierte Darstellung der Ergebnisse der Umfrage 2003 erfolgt im Laufe des Jahres 2004.

Abbildung 2

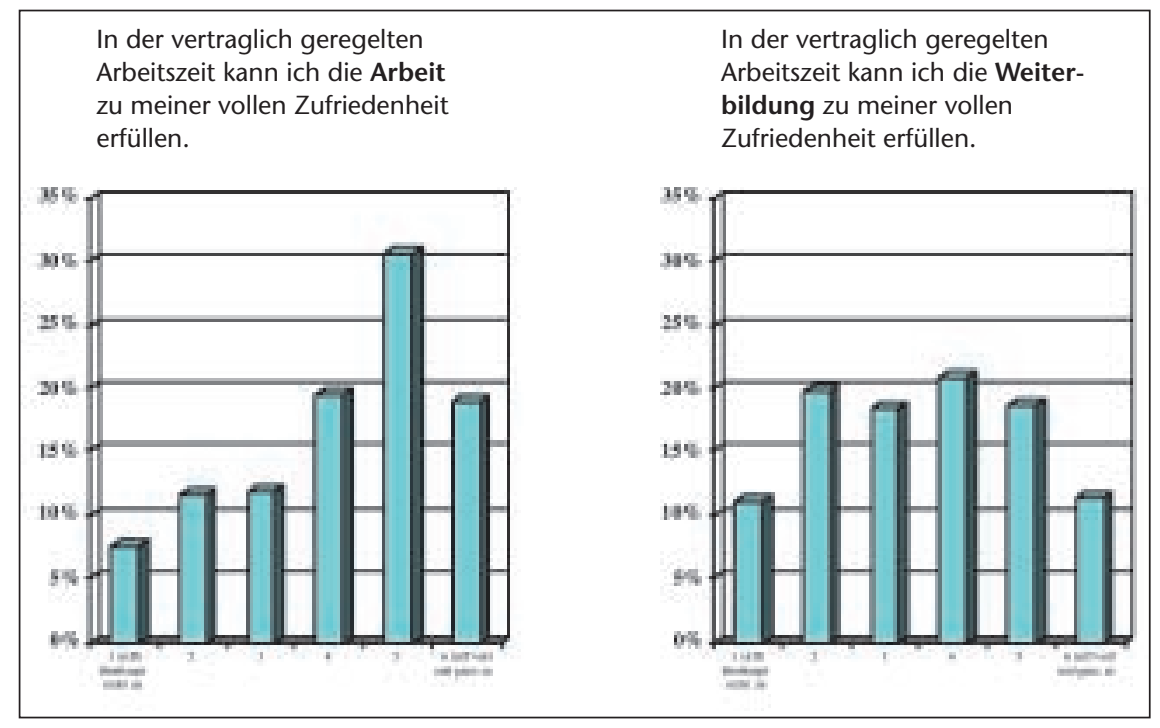

